\title{
Sur les réarrangements de fonctions de la classe $A$
}

par

JEAN-PIERRE KAHANE (Orsay)

En hommage

à MM. S. Mazur et W. Orlicz

1. Soit $I$ un intervalle fermé borné sur la droite. On désigne par $A(I)$ l'ensemble des fonctions continues sur $I$ et prolongeables sur la droite en transformées de Fourier de fonctions sommables. Si $I$ a une longueur strictement inférieure à $l, A(I)$ est aussi bien l'ensemble des fonctions continues sur $I$ et prolongeables en fonctions $l$-périodiques dont la série de Fourier est absolument convergente.

On appellera dans cet article réarrangement d'une fonction $g$ continue sur $I$ toute fonction go $\varphi$, composée de $g$ et d'un homéomorphisme $\varphi$ de $I$. On peut demander s'il existe des fonctions $f$, continues sur $I$, dont aucun réarrangement n'appartienne à $A(I)$; c'est là un problème, toujours ouvert, posé par Lusin ([1], p. 168). On peut aussi demander si les réarrangements des fonctions de la classe $A(I)$ engendrent (par combinaisons linéaires) l'espace vectoriel $C(I)$ des fonctions continues sur $I$; les théorèmes ci-dessous répondent affirmativement à cette question.

Supposons donnée une fonction $h(t) \geqslant 0$ sur $[0, \infty]$, croissante (au sens large), sous-additive $\left(h\left(t+t^{\prime}\right) \leqslant h(t)+h\left(t^{\prime}\right)\right)$ et telle que

$$
\varlimsup_{t \rightarrow 0} \frac{h(t)}{t}=\infty \text {. }
$$

On désigne par $H$ l'ensemble des fonctions $\varphi$ croissantes (au sens large) et continues appliquant $I$ sur $I$, pour lesquelles

$$
\lim _{\left|t-t^{\prime}\right| \rightarrow 0} \frac{\left|\varphi(t)-\varphi\left(t^{\prime}\right)\right|}{h\left(\left|t-t^{\prime}\right|\right)}=0 .
$$

$H$ est un convexe fermé de l'espace de Banach $\lambda_{h}(I)$ constitué par les fonctions $\varphi$ de $O(I)$ qui vérifient (2), la norme étant

$$
\|\varphi\|_{\lambda_{h}(I)}=\sup _{t, t^{\prime}} \frac{\left|\varphi(t)-\varphi\left(t^{\prime}\right)\right|}{h\left(\left|t-t^{\prime}\right|\right)}+\sup _{t}|\varphi(t)| .
$$


THÉORÈMe 1. En dehors d'un ensemble de première catégorie sur $H \times H$, tout couple $\left(\varphi_{1}, \varphi_{2}\right)$ d'étéments de $\mathrm{H}$ jouit de la propriété suivante: toute fonction $f$ de $C(I)$ s'écrit

$$
f=g_{1} \circ \varphi_{1}+g_{2} \circ \varphi_{2}
$$

pour un choix convenable de $g_{1}$ et de $g_{2}$ dans $A(I)$.

THÉoRł̀mE 2. En dehors d'un ensemble de première catégorie sur $H \times H \times H$, tout triplet $\left(\varphi_{1}, \varphi_{2}, \varphi_{3}\right)$ d'éléments de $H$ jouit de la propriété suivante: toute fonotion $f$ de $C(I)$ s'écrit

$$
f=g \circ \varphi_{1}+g \circ \varphi_{2}+g \circ \varphi_{3}
$$

pour un choix convenable de $g$ dans $A(I)$.

Ces énoncés sont valables aussi bien pour des fonctions à valeurs complexes que pour des fonctions à valeurs réelles. Il suffit évidemment de les démontrer dans ce dernier cas.

On donnera à la fin de l'article quelques compléments et commentaires.

2. Les démonstrations s'appuient sur les deux lemmes suivants.

LEMME 1. Soit $\varphi$ une fonction de $H, k$ un entier $\geqslant 1, \delta$ un nombre positif; $S$ une partie de I formée d'intervalles dont la longueur est comprise entre $\delta$ et $k \delta$, séparés par des intervalles de longueur $\delta$; et enfin $\psi$ une fonction de $H$, localement constante sur $S$, linéaire dans les intervalles contigus à $S$, et telle que

Alors

$$
\sup _{t}|\psi(t)-\varphi(t)| \leqslant \omega_{\varphi}(k \delta)=\sup _{\left|t-t^{\prime}\right| \leqslant k \delta}\left|\varphi(t)-\varphi\left(t^{\prime}\right)\right| .
$$

$$
\|\psi-\varphi\|_{\lambda_{h}(I)} \leqslant \varepsilon(\varphi, k, \delta)
$$

$\varepsilon(\varphi, k, \delta)$ ne dépendant que de $\varphi, k$, et $\delta$, et tendant vers zéro quand $\delta \rightarrow 0$.

La preuve est immédiate à partir de (3) et des inégalités qui en découlent, c'est-à-dire

$$
\left|\psi(t)-\psi\left(t^{\prime}\right)-\varphi(t)+\varphi\left(t^{\prime}\right)\right| \leqslant 2 \omega_{\varphi}(k \delta),
$$

que l'on utilise quand $\left|t-t^{\prime}\right|>\delta$, et

$$
\left|\psi(t)-\psi\left(t^{\prime}\right)-\varphi(t)+\varphi\left(t^{\prime}\right)\right| \leqslant \frac{2\left|t^{\prime}-t\right|}{\delta} \omega_{\varphi}(k \delta)+\omega_{\varphi}\left(\left|t-t^{\prime}\right|\right),
$$

valable pour $\left|t-t^{\prime}\right| \leqslant \delta$.

LeMre 2. Soit $t_{1}, t_{2}, \ldots, t_{N}$ une suite croissante de points de $I$, linéairement indépendants sur les rationnets, et soit $\varepsilon_{1}, \varepsilon_{2}, \ldots, \varepsilon_{N}$ une suite $\grave{a}$ valeurs $-1,0$ ou +1 . Il existe une fonction $g$ de la classe $A(\boldsymbol{R})$ (c'est$\grave{a}$-dire transformée de Fourier de fonction sommable) ayant les propriétés suivantes:
(a) $\|g\|_{A(R)}=\|\hat{g}\|_{L^{1}(\boldsymbol{R})} \leqslant 2$;
(b) $g\left(t_{n}\right)=\frac{1}{4} \varepsilon_{n}(n=1,2, \ldots, N)$;

(c) $\|g\|_{C_{(I)}} \leqslant \frac{1}{4}$

(d) $g(t) \geqslant-\frac{1}{8}$ (resp. $\left.g(t) \leqslant \frac{1}{8}\right)$ sur tout intervalle $\left[t_{n}, t_{n+1}\right]$ pour lequel $\varepsilon_{n} \geqslant 0, \varepsilon_{n+1} \geqslant 0$ (resp. $\varepsilon_{n} \leqslant 0, \varepsilon_{n+1} \leqslant 0$ ).

Preuve. Quitte à faire une translation de l'origine, on peut supposer qu'aucun des $t_{n}$ n'est nul. Alors, pour $\eta>0$ assez petit, la fonction $g$ dont la transformée de Fourier est

$$
\hat{g}(u)=\frac{\sin ^{2} \eta u}{2 \eta u^{2}}\left(\prod_{n=1}^{N}\left(1+\varepsilon_{n} \cos t_{n} u\right)-1\right)
$$

satisfait aux conditions (a), (b), (c) et (d), comme on s'en convainc assez facilement. On a même, au lieu de (c), $\|g\|_{L^{\infty}(R)} \leqslant \frac{1}{4}$.

En vue du théorème 1, on commence par la proposition suivante:

Proposition 1. Soit $f \in C(I)$. Désignons par F l'ensemble des couples $\left(\varphi_{1}, \varphi_{2}\right)$ de $H \times H$ tels que, pour tout couple de fonctions $g_{1}$ et $g_{2}$ de $A(I)$, de normes $\leqslant 2\|f\|_{C_{(I)}}$ dans $A(I)$, on ait

$$
\left\|f-g_{1} \circ \varphi_{1}-g_{2} \circ \varphi_{2}\right\|_{C_{(I)}} \geqslant \frac{8}{9}\|f\|_{C_{(I)}} .
$$

Sauf si $f$ est identiquement nul, $F$ est un fermé non dense dans $H \times H$.

Démonstration. On peut sans restriction supposer $\|f\|_{C_{(I)}}=1$. $F$ est visiblement un fermé. Montrons qu'il est non dense. Pour cela, nous allons montrer que dans tout voisinage d'un $\left(\varphi_{1}, \varphi_{2}\right)$ donné de $F$, il existe un $\left(\psi_{1}, \psi_{2}\right)$ de $H \times H$ tel que, pour un choix convenable de $g_{1}$ et de $g_{2}$ dans la boule $\|g\| \leqslant 2$ de $A(I)$, on ait

$$
\left\|f-g_{1} \circ \psi_{1}-g_{2} \circ \psi_{2}\right\|_{(I)} \leqslant \frac{7}{8} .
$$

Soit $S_{1}$ et $S_{2}$ deux parties de $I$ disjointes, constituées d'intervallec (ouverts, semi-ouverts ou fermés) de même longueur $\delta$, telles que $S_{1} \cup S_{2}=I ; S_{1}$ et $S_{2}$ sont des ensembles du type $S$ décrit au lemme 1, aves $k=1$. Le couple $\left(\varphi_{1}, \varphi_{2}\right)$ étant donné, soit $\psi_{1}$ et $\psi_{2}$ deux fonctions de $B$, définies respectivement à partir de $\varphi_{1}, S_{1}$ et $\varphi_{2}, S_{2}$ comme la fonction $\psi$ du lemme 1 à partir de $\varphi, S$, avec la condition additionnelle que $\psi_{1}$ (resp. $\psi_{2}$ ) prend des valeurs indépendantes sur les différents intervalles constituant $S_{1}$ (resp. $\left.S_{2}\right)$.

Soit $s_{i, 1}, s_{i, 2}, \ldots, s_{i, N}$ les intervalles de $S_{i}(j=1,2)$, ordonnés de gauche à droite. Posons $\psi_{j}\left(s_{j, n}\right)=t_{j, n}=t_{n}$ et

$$
\varepsilon_{j, n}=\varepsilon_{n}=\left\{\begin{aligned}
1 & \text { si } f \geqslant 0 \text { sur } s_{j, n} \\
-1 & \text { si } f \leqslant 0 \text { sur } s_{j, n} \\
0 & \text { sinon }
\end{aligned}\right.
$$


En appliquant le lemme 2 , on a une fonction $g_{j}$ de norme $\leqslant 2$ dans $A(R)$, donc (restreinte à $I$ ) de norme $\leqslant 2$ dans $A(I)$, vérifiant (b), (c), (d).

Si $\delta$ est assez petit, $\left(\psi_{1}, \psi_{2}\right)$ est arbitrairement proche de $\left(\varphi_{1}, \varphi_{2}\right)$ dans $H \times H$ (lemme 1 ). On peut supposer d'autre part $\omega_{f}(\delta) \leqslant \frac{1}{8}$. Montrons alors que (4) a lieu.

Soit $x$ un point de $I$. Supposons, pour fixer les idées, $x \in S_{1}$. Soit $s_{1}=s_{1, n+1}$ l'intervalle de $S_{1}$ qui contient $x, s_{2, n}$ et $s_{2, n+1}$ les intervalles de $S_{2}$ adjacents à $s_{1}$.

Si $|f(x)| \leqslant \frac{3}{8}$, on a, d'après la condition (c),

$$
\left|f(x)-g_{1}\left(\varphi_{1}(x)\right)-g_{2}\left(\varphi_{2}(x)\right)\right| \leqslant \frac{3}{8}+\frac{1}{2}=\frac{7}{8} .
$$

Si $f(x) \geqslant \frac{3}{8}$, la condition sur $\omega_{f}(\delta)$ entraîne $f \geqslant 0$ sur $s_{1}$, sur $s_{2, n}$ et sur $s_{2, n+1}$, donc $\varepsilon_{1, n+1}=\varepsilon_{2, n}=\varepsilon_{2, n+1}=1$. La condition (b), appliquée à $g_{1}$, donne

$$
g_{1}\left(\varphi_{1}(x)\right)=g_{1}\left(t_{1, n+1}\right)=\frac{1}{4} .
$$

La condition (d) est appliquable à $g_{2}$ et, puisque $\varphi_{2}$ est croissante, $\varphi_{2}(x)$ est contenu dans l'intervalle $\left[t_{2, n}, t_{2, n+1}\right]$; donc

$$
g_{2}\left(\varphi_{2}(x)\right) \geqslant-\frac{1}{8} \text {. }
$$

Il en résulte que

$$
f(x)-g_{1}\left(\varphi_{1}(x)\right)-g_{2}\left(\varphi_{2}(x)\right) \leqslant 1-\frac{1}{4}+\frac{1}{8}=\frac{7}{8} .
$$

D'autre part, la condition (c) entraîne

$$
f(x)-g_{1}\left(\varphi_{1}(x)\right)-g_{2}\left(\varphi_{2}(x)\right) \geqslant-\frac{1}{2} \text {. }
$$

En changeant les signes, on traite de même le cas $-f(x) \geqslant \frac{3}{3}$. Done (4) a lieu, ce qui achève la démonstration de la proposition 1.

Démonstration $d u$ théorème 1 . Soit $f_{(k)}(k=1,2, \ldots)$ une suite dense dans $C(I)$, et $F_{(k)}$ la suite correspondante de fermés non denses de $H \times H$. La réunion des $F_{(k)}$ est l'ensemble exceptionnel $\Phi$, qui est de première catégorie. Si $\left(\varphi_{1}, \varphi_{2}\right) \notin \Phi$ et $f \epsilon C(I)$, il est aisé de voir qu'il existe deux fonctions $g_{1}$ et $g_{2}$, de normes $\leqslant 2\|f\|_{C(I)}$ dans $A(I)$, et telles que

$$
\left\|f-g_{1} \circ \varphi_{1}-g_{2} \circ \varphi_{2}\right\|_{C_{(I)}} \leqslant \frac{9}{10}\|f\|_{C_{(I)}} .
$$

Fixons $\left(\varphi_{1}, \varphi_{2}\right) \notin \Phi$. Désignons par $\gamma_{1}(f)$ et $\gamma_{2}(f)$ les fonctions $g_{1}$ et $g_{2}$ qui viennent d'être définies. Définissons par récurrence $f_{0}=f$, élément donné de $C(I)$,

$$
f_{m+1}=f_{m}-\gamma_{1}\left(f_{m}\right) \circ \varphi_{1}-\gamma_{2}\left(f_{m}\right) \circ \varphi_{2}
$$

On a, en supposant $\|f\|_{C(I)}=1$,

$$
\left\|f_{m}\right\|_{C(I)} \leqslant\left(\frac{9}{10}\right)^{m}, \quad\left\|\gamma_{j}\left(f_{m}\right)\right\|_{A(I)} \leqslant 2\left(\frac{9}{10}\right)^{m} .
$$

Les séries $\sum_{m=0}^{\infty} \gamma_{j}\left(f_{m}\right), j=1,2$, sont done convergentes dans $A(I)$, et leurs sommes $g_{1}^{*}$ et $g_{2}^{*}$ vérifient

$$
f-g_{1}^{*} \circ \varphi_{1}-g_{2}^{*} \circ \varphi_{2}=0 .
$$

Cela achève la démonstration du théorème 1.

Démonstration du théorème 2. En vue du théorème 2, on remplace la proposition 1 par la suivante:

Propositron 2. Soit $f \epsilon C(I)$. Soit $F$ l'ensemble des triplets $\left(\varphi_{1}, \varphi_{2}, \varphi_{3}\right)$ de $H \times H \times H$ tels que, pour toute fonction $g$ de $A(I)$, de norme $\leqslant 2\|f\|_{C_{(I)}}$ dans $A(I)$, on ait

$$
\left\|f-g \circ \varphi_{1}-g \circ \varphi_{2}-g \circ \varphi_{3}\right\|_{C(I)} \geqslant \frac{8}{9}\|f\|_{C(I)} .
$$

Sauf sif est identiquement nul, $F$ est un fermé non dense dans $H \times H \times H$.

La démonstration est très voisine de celle de la proposition 1. En identifiant l'origine et l'extrémité de $I$, on définit $S_{1}, S_{2}, S_{\mathbf{2}}$ comme trois insembles fermés, se déduisant l'un de l'autre par les translations $\pm \delta$, et formés d'intervalles fermés de longueur commune $2 \delta$ séparés par des entervalles ouverts de longueur $\delta$. Chaque point de $I$ appartient à deux au moins de ces trois ensembles. Le triplet $\left(\varphi_{1}, \varphi_{2}, \varphi_{3}\right)$ étant donné, on définit $\left(\psi_{1}, \psi_{2}, \psi_{3}\right)$ comme dans le lemme 1 , avec la condition additionnelle que les valeurs prises par $\psi_{1}$ sur les intervalles constituant $S_{1}$, par $\psi_{2}$ sur les intervalles constituant $S_{2}$ et par $\psi_{3}$ sur les intervalles constituant $S_{3}$ sont indépendantes sur les rationnels.

Le lemme 2 s'applique alors en prenant pour $t_{1}, t_{2}, \ldots, t_{N}$ la suite des valeurs prises par $\psi_{1}$ sur $S_{1}, \psi_{2} \operatorname{sur} S_{2}, \psi_{3} \operatorname{sur} S_{3}$. Si $t_{n}=\psi_{j}(s), s$ étant l'un des intervalles constituant $S_{j}$, on pose

$$
\varepsilon_{n}=\left\{\begin{aligned}
1 & \text { si } f \geqslant 0 \text { sur } s \\
-1 & \text { si } f \leqslant 0 \text { sur } s \\
0 & \text { sinon. }
\end{aligned}\right.
$$

Sous la condition $\omega_{f}(2 \delta) \leqslant \frac{1}{8}$, la fonction $g$ ainsi définie satisfait pour tout $x$ à l'inégalité

$$
\left|f(x)-g\left(\psi_{1}(x)\right)-g\left(\psi_{2}(x)\right)-g\left(\psi_{3}(x)\right)\right| \leqslant \frac{7}{8}\|f\|_{C_{(I)}} .
$$


On s'en assure aisément en distinguant les cas $|f(x)| \leqslant \frac{1}{8}$ (l'inégalité résultant immédiatement de (c)) et $|f(x)| \geqslant \frac{1}{8}$ (l'inégalité résultant alors de (b) et (c)). On n'utilise pas la condition (d) du lemme 2.

La proposition 2 est ainsi établie.

La démonstration du théorème 2 est calquée sur celle du théorème 1 , en utilisant la proposition 2 au lieu de la proposition 1.

3. Les démonstrations qui précèdent sont inspirées pour une part de la méthode utilisée par R. Kaufman pour mettre en évidence des ensembles de Kronecker [3], d'autre part de la version donnée par G. Lorentz du théorème de superposition de Kolmogorov ([4], chapitre XI). Elles s'appliquent dans des cas un peu plus étendus: par exemple, on peut considérer différentes fonctions $h_{1}, h_{2}, h_{3}$ au lieu d'une seule fonction $h$, définir en conséquence $H_{1}, H_{2}, H_{3}$, et considérer les $\left(\varphi_{1}, \varphi_{2}\right)$ dans $H_{1} \times H_{2}$ et les $\left(\varphi_{1}, \varphi_{2}, \varphi_{3}\right)$ dans $H_{1} \times H_{2} \times H_{3}$.

On peut également, en combinant les deux démonstrations, avoir l'énoncé suivant: sauf sur un ensemble de première catégorie dans $H_{1} \times H_{2} \times$ $\times H_{3}$, tout triplet $\left(\varphi_{1}, \varphi_{2}, \varphi_{3}\right)$ de $H_{1} \times H_{2} \times H_{3}$ a la propriété que toute fonction $f$ de $C(I)$ s'écrive

$$
f=g_{1} \circ \varphi_{1}+g_{2} \circ \varphi_{2}=g \circ \varphi_{1}+g \circ \varphi_{2}+g \circ \varphi_{3}
$$

pour un choix convenable des fonctions $g_{1}, g_{2}$ et $g$ dans $A(I)$.

On peut d'autre part, à la place de l'intervalle $I$, considérer le cercle $T$ ou la droite $\boldsymbol{R}$, et définir en conséquence les classes $H$ d'homéomorphismes de $\boldsymbol{T}$ ou $\boldsymbol{R}$. Le théorème 1 et le théorème 2 s'adaptent mot pour mot ainsi, que la dernière remarque. En particulier, il existe des homéomorphismes $\varphi_{1}, \varphi_{2}, \varphi_{3}$ de $\boldsymbol{T}$ (resp. $\boldsymbol{R}$ ) tels que toute fonction $f$ continue sur $\boldsymbol{T}$ (resp. continue et nulle à l'infini sur $\boldsymbol{R})$ s'écrive

$$
f=g_{1} \circ \varphi_{1}+g_{2} \circ \varphi_{2}=g \circ \varphi_{1}+g \circ \varphi_{2}+g \circ \varphi_{3}
$$

pour un choix convenable de $g_{1}, g_{2}, g$ dans $A(\boldsymbol{T})$ (resp. $\left.A(\boldsymbol{R})\right), A(\boldsymbol{T})$ désignant l'ensemble des fonctions continues sur $\boldsymbol{T}$ à séries de Fourier absolument convergentes, et $\boldsymbol{A}(\boldsymbol{R})$, comme on l'a vu, l'ensemble des transformées de Fourier des fonctions sommables.

Si l'on considère un ensemble fermé totalement discontinu $E$ contenu dans $I$, on a le résultat suivant: sauf si $\varphi$ est pris dans un ensemble de première catégorie dans $H$, toute fonction $f$ continue sur $\varphi(E)$ s'écrit $f=g \circ \varphi, g$ étant la restriction à $\varphi(E)$ d'une fonction de la classe $A$. Lorsque cette condition est réalisée, on dit que $\varphi(E)$ est un ensemble de Helson. On a même un résultat plus fort: sauf si $\varphi$ est pris dans un ensemble de première catégorie dans $H, \varphi(E)$ est un ensemble de Kronecker [3].

Comme corollaire du théorème 1 , on voit que, sauf le cas exceptionnel, l'ensemble image par $\varphi_{1}$ ou $\varphi_{2}$ de l'ensemble des zéros de $\varphi_{1}-\varphi_{2}$ est un ensemble de Helson. En d'autres termes, sauf si $\left(\varphi_{1}, \varphi_{2}\right)$ est pris dans un ensemble de première catégorie dans $H \times H$, l'ensemble des points invariants de la fonction $\varphi_{1} \circ \varphi_{2}^{-1}$ (où $\varphi_{2}^{-1}$ est la fonction réciproque de $\varphi_{2}$ ) est un ensemble de Helson.

On peut se demander si les théorèmes 1 et 2 ne sont pas des cas particuliers d'un théorème plus général, dans lequel la conclusion serait: toute fonction $f$ de $O(I)$ s'écrit

$$
f=g \circ \varphi_{1}+g \circ \varphi_{2}
$$

pour un choix convenable de $g$ dans $A(I)$. $\Pi$ n'en est rien. En effet, si l'on pouvait écrire toute fonction $h$ de $C(I)$ sous la forme $g+g \circ \varphi$, où $q$ est un homéomorphisme croissant de $I$ donné une fois pour toutes, et $g$ une fonction de $A(I)$, l'application $g \rightarrow g+g \circ \varphi$ serait bijective de $A(I)$ dans $C(I)$ (on vérifie facilement que $g=-g \circ \varphi=g \circ \varphi \circ \varphi$ entraîne $g=0$ ); et cela est impossible, d'après un théorème de Banach, puisque les normes \|\|$_{A(I)}$ et \|\|$_{C_{(I)}}$ ne sont pas équivalentes.

Des énoncés voisins des théorèmes 1 et 2 , concernant les réarrangements des suites de coefficients de Fourier, ont été publiés dans [2].

\section{Travaux cités}

[1] Н. Бар и, Тригонометрические ряды, Москва 1961.

[2] J.-P. Kahane, Sur les réarrangements des suites de coefficients de Fourier-Lebesgue, C. R. Acad. Sci. Paris 265 (1967), p. $310-312$.

[3] R. Kaufman, A functional method for linear sets, Israel J. Mathematies 5 (1967), p. $185-187$.

[4] G. Lorentz, Approximation of functions, New York 1967.

Ręu par la Rédaction le 5. 3. 1968 